\title{
Chemometric strategies for enhancing the chromatographic methodologies with second-order data analysis of compounds when peaks are overlapped
}

\author{
H.C. Goicoechea ${ }^{a^{*}}$, M.J. Culzoni ${ }^{a}$, M.D. Gil Garcia ${ }^{\mathrm{b}}$, M. Martinez Galera ${ }^{\mathrm{b}}$ \\ a, Laboratorio de Desarrollo Analítico y Quimiometría (LADAQ), Cátedra de Química Analítica I, Facultad \\ de Bioquímica y Ciencias Biológicas, Universidad Nacional del Litoral, Ciudad Universitaria, Santa Fe \\ S3000ZAA, Argentina, \\ ${ }^{b}$ Department of Hydrogeology and Analytical Chemistry, University of Almeria, La Cañada de San Urbano \\ 04120 Almeria, Spain
}

\begin{abstract}
This overview covers the different chemometric strategies linked to chromatographic methodologies that have been used and presented in the recent literature to cope with problems related to incomplete separation, the presence of unexpected components in the sample, matrix effect and changes in the analytical signal due to pre-treatment of sample.

Among the different chemometric strategies it focuses on pre-treatment of data to correct background and time shift of chromatographic peaks and the use of second-order algorithms to cope with overlapping peaks from analytes or from analytes and interferences in liquid chromatography coupled to diode array, fast-scanning fluorescence spectroscopy and mass spectrometry detectors. Finally the review presents the strategies used to deal with changes in the analytical response as result of matrix effect in liquid and gas chromatography, as well as the use of standardization strategies to correct modifications in the analytical signal as a consequence of sample pre-treatment in liquid chromatography.
\end{abstract}

Keywords: Chromatography, Chemometrics, Second-order data, Signal pre-treatment

*Corresponding author: H.C. Goicoechea (e-mail: hgoico@fbcb.unl.edu.ar) M. Martínez Galera (e-mail: mmartine@ual.es) 


\section{Introduction}

Developing a chromatograpic method generally implies optimizing the experimental conditions, in order to guarantee complete separation of all sample components [1]. In chromatography, the retention factor $(\mathrm{k})$ is the degree of retention of the sample component in the column. In most chromatographic analysis, analytes elute with retention factors between 1 and 20 allowing their complete separation. A peak with $\mathrm{k}$ equal to 0 is a component that does not interact with the stationary phase and elutes in the void volume [2]. Chromatographic separations can become a difficult task when complex samples have to be analyzed. The main drawbacks involved in handling complex samples are that the nature and the amount of the co-eluting matrix compounds may be rather variable between samples, in such a way that matrix effects in a series of samples can also be highly variable and difficult to predict [3]. Nevertheless, the use of chemometrics may provide a useful resource for accurate analyte quantitation when the complete separation is not accomplished or new compounds are present in the sample being analyzed [4]. Chemometrics is especially useful in chromatography when second-order data are recorded, for example, using a diode-array detector (DAD), a fast-scanning luminescence or a mass spectrometry based (MS) detector during the chromatographic time evolution. An interesting intrinsic property that secondorder data may show (if they are modeled with convenient second-order algorithms) is the so-called "second-order advantage" [5], which in principle permits analyte quantitation in samples containing unexpected components, i.e., compounds not included in the calibration set [6]. This fact allows one to build a predictive model with a limited number of standards, yet quantitating the analyte in the presence of potential interferents [6].

The use of second-order multivariate algorithms has been shown to play a critical role in several analytical fields, as can be gathered from a literature survey in relevant analytical, chemometrics and applied journals [6,7]. Specifically, an important number of reports have been presented ocusing on the resolution of really complex samples using liquid chromatography and exploiting the mentioned second-order advantage [8-12]. In this context, extremely important issues such as reduction in the time of analysis and consequently costs and amount of contaminant solvents should be considered [12].

Several algorithms can be cited among the approaches involving the second-order advantage: generalized rank annihilation (GRAM) [13], direct trilinear decomposition (DTLD) [14,15], selfweighted alternating trilinear decomposition (SWATLD) [16], alternating penalty trilinear decomposition (APTLD) [17], parallel factor analysis (PARAFAC) [18], multivariate curve resolution alternating least squares (MCR-ALS) [19], and the most recently implemented bilinear 
least squares (BLLS) [20], unfolded partial least squares/residual bilinearization (U-PLS/RBL) [21] and artificial neural networks followed by residual bilinearization (ANN/RBL) [22].

Interestingly, a k value lower than 1 does not result in a differential migration of the component and originates dissimilarities in both times of elution and peak shapes, leading to data without the property of trilinearity. Second-order data are trilinear when each compound in all experiments treated together can be described by a triad of invariant pure profiles [23]. In chromatography with DAD detector, each analyte should have the same time and spectral profile in all the samples and only differs in the amount in which it intervenes. In this situation, algorithms such as MCR-ALS (which can solve this type of problems by resorting to the mathematical resource of matrix augmentation) and PARAFAC2, a variant of PARAFAC allowing for distinct time profiles in each experimental sample [24], have been proved to be useful alternatives for treating these data since they are more flexible with regard to trilinearity $[25,26]$. However, when data are conveniently pretreated in order to alleviate the above-mentioned problems, good results can be obtained using GRAM, PARAFAC or RBL based algorithms [10,27].

In the present review we describe different chemometric strategies that have been used and presented in recent literature reports to cope with the problem of incomplete separation, especially, when non-modeled components appears in the sample.

\section{Pre-treatments of data}

In a previous paper, Daszykowski and Walczak [4] pointed out that chromatographic performance can be enhanced by eliminating noise and background components, thus becoming the chromatogram baseline elimination a crucial step for reducing both the complexity and the number of the unexpected components. Moreover, it was demonstrated that the use of signal pre-treatments such as baseline and time shift corrections improve the quality of second-order chromatographic signals and, as a consequence, the performance of resolution by second-order algorithms [9-11,28]. In the present section, different strategies which were developed with these aims and reported in the literature will be revised.

\subsection{Background correction}

A proper pre-processing step is crucial to determine the quality of chromatograms, influencing the final results of chromatographic analysis. Chromatograms (as any other instrumental signal) contain three major components: signal, noise and background, which differ in their frequency [4]. Signal enhancement can be achieved by eliminating noise and background components. As was stated previously, elimination of the chromatogram baseline could result in a critical step for reducing complexity of the analytical task. There are several algorithms that can be useful to 
overcome this problem, the former attempts probably being made by Cecil and Rutan [29]. They worked with fluorescence detection in liquid chromatography with an intensified diode-array detector and the data analysis methods used included Kalman filter-based methods for adaptive subtraction of background responses, shift correction and linear regression analysis of overlapped responses. After that, a new procedure for detecting and correcting baseline offset/drift and spectral background in hyphenated chromatographic data was presented by Brereton and co-workers [30], which was based on congruence analysis and least-squares fit of the zero-component regions. The procedure consists of several different steps: first, the major principal components in the zerocomponent chromatographic regions are extracted before the appearance of the first eluting chemical constituent and after elution of the last chemical constituent in a peak cluster. Then, comparison of the loading patterns of the first principal component in the two zero-component regions by means of congruence analysis was used to reveal the presence of a constant spectral background and/or systematic baseline offset or drifts. If baseline drift is revealed, the baseline for the whole chromatogram is estimated by means of a least-squares fit of the data from the two zerocomponent regions with retention time as 'independent' variable. A background-corrected chromatogram is finally obtained by subtracting the estimated spectral background and the estimated baseline from the original data.

Recently, with the increasing of the use of second-order data, several algorithms have been proposed. One of them is the methodology presented by Eilers, i.e., the asymmetric least-squares method [31], which was recently adapted to multidimensional data [32]. This method consists in the matrix background estimation $\mathbf{F}(\mathrm{J} \times \mathrm{K})$ from the data matrix $\mathbf{M}(\mathrm{J} \times \mathrm{K})$, where $\mathbf{J}$ is the number of digitized wavelengths and $\mathrm{K}$ the number of migration times. For achieving this purpose, a $\mathbf{B} 1(\mathrm{~L} \times \mathrm{J})$ spline basis matrix along the rows of the $\mathbf{M}$ matrix and a $\mathbf{B} 2(\mathbf{M} \times \mathrm{K})$ spline basis matrix along the columns of the $\mathbf{M}$ matrix are used. Generally, the literature [33] suggests a compromise of 10 basis function, i.e., $\mathrm{L}=\mathrm{M}=10$. $\mathbf{F}$ can be represented as:

$$
f_{J, K}=\sum_{L, M} b 1_{L J} b 2_{M K} a_{L M}
$$

where $\mathrm{a}_{\mathrm{LM}}$ is the (L,M) element of a matrix A containing the regression coefficients, which can be calculated by minimizing the following cost function:

$$
Q=\sum_{L, M} v_{J K} \boldsymbol{\varphi}_{J K}-f_{J K}^{\stackrel{2}{\rightleftharpoons}}+p
$$

where $\mathrm{y}$ is the experimental signal, $\mathrm{f}$ a smooth trend (the baseline approximation), and $\mathrm{v}$ are the prior weights. The elements of $\mathrm{v}$ should have large values in the parts of the signal where it is allowed to affect estimation of the baseline. Consider the following choice of asymmetric weights: 
$\mathrm{v}_{\mathrm{JK}}=\mathrm{p}$ if $\mathrm{v}_{\mathrm{JK}}>\mathrm{f}_{\mathrm{JK}}$ and $\mathrm{v}_{\mathrm{JK}}=1-\mathrm{p}$ if $\mathrm{v}_{\mathrm{JK}} \leq \mathrm{f}_{\mathrm{JK}}$ with $0<\mathrm{p}<1$. Positive deviations from the trend will result in weights different from negative residuals. Experience shows that starting from $v \cong 1$, and iterating between the two computations, quickly and reliably leads to a solution in about 10 iterations. Finally, in Eq. (2) there is a penalty term defined by:

$$
P=\left[\sum_{L}\left(a_{1}^{d} a_{L}{ }^{2}+\sum_{L}\left(a_{2}^{d} a_{M}\right)^{2}\right]\right.
$$

where $\Delta_{1}$ and $\Delta_{2}$ are differences of order d calculated for each column of $\mathbf{A}\left(\mathbf{a}_{\mathrm{L}}\right)$ and each row of $\mathbf{A}$ $\left(\mathbf{a}_{\mathrm{M}}\right)$, respectively. As can be seen in Eq. (3), if different values are used for the regularization parameter $\lambda$, the penalty may have different influences for vertical and horizontal directions. As an example, Fig. 1A shows an original 3D chromatogram corresponding to the determination of 11 pharmaceuticals in river water (for more details see Ref. [11]). The landscape corresponds to a data matrix M (40×302), which was obtained with a HPLC-DAD system, using a wavelength range between 200 and $350 \mathrm{~nm}$. As can be appreciated in this figure, a significant baseline is originated during the chromatographic procedure. The matrix background estimation $\mathbf{F}(40 \times 302)$ by implementing the asymmetric least-squares method can be seen in Fig. 1B. This matrix was obtained setting $\mathrm{L}=\mathrm{M}=10$ and an asymmetry parameter $(\mathrm{p}=0.005)$ [see Eq. (2)]. The subtraction of $\mathbf{F}$ matrix to $\mathbf{M}$ matrix furnishes the corrected matrix, whose representation can be observed in Fig. 1C. Finally, in order to have a better visualization of the effect of base line subtraction, the chromatograms registered a $245 \mathrm{~nm}$ are represented in Fig. 2.

On the other hand, on-line coupling between LC and FT-IR becomes a difficult task: as the mobile phases employed in LC absorb strongly in the mid infrared, their accurate compensation is crucial to obtain characteristic analyte spectra. In the case of on-line isocratic LCFT-IR systems, correction for mobile phase absorption can be carried out by subtracting the spectra of the eluent recorded at the beginning of the run or immediately before elution of the analytes of interest from the spectra when the analyte elutes.

However, when using the gradient technique, accurate background correction presents important difficulties because of existing changes in intensity and shape of the eluent absorption bands, which may be up to several orders of magnitude more intense than the absorption due to analytes. Different chemometric techniques have been proposed to overcome this problem. Among them, a method named objective subtraction of solvent spectrum has been proposed by Istva'n along with iterative use of PARAFAC and PARAFAC2 which yielded promising results when analyzing isocratic LC-IR data sets [34,35]. Afterward, univariate and multivariate methods have been developed by Quintas et al. to perform eluent subtraction in continuous liquid flow systems under 

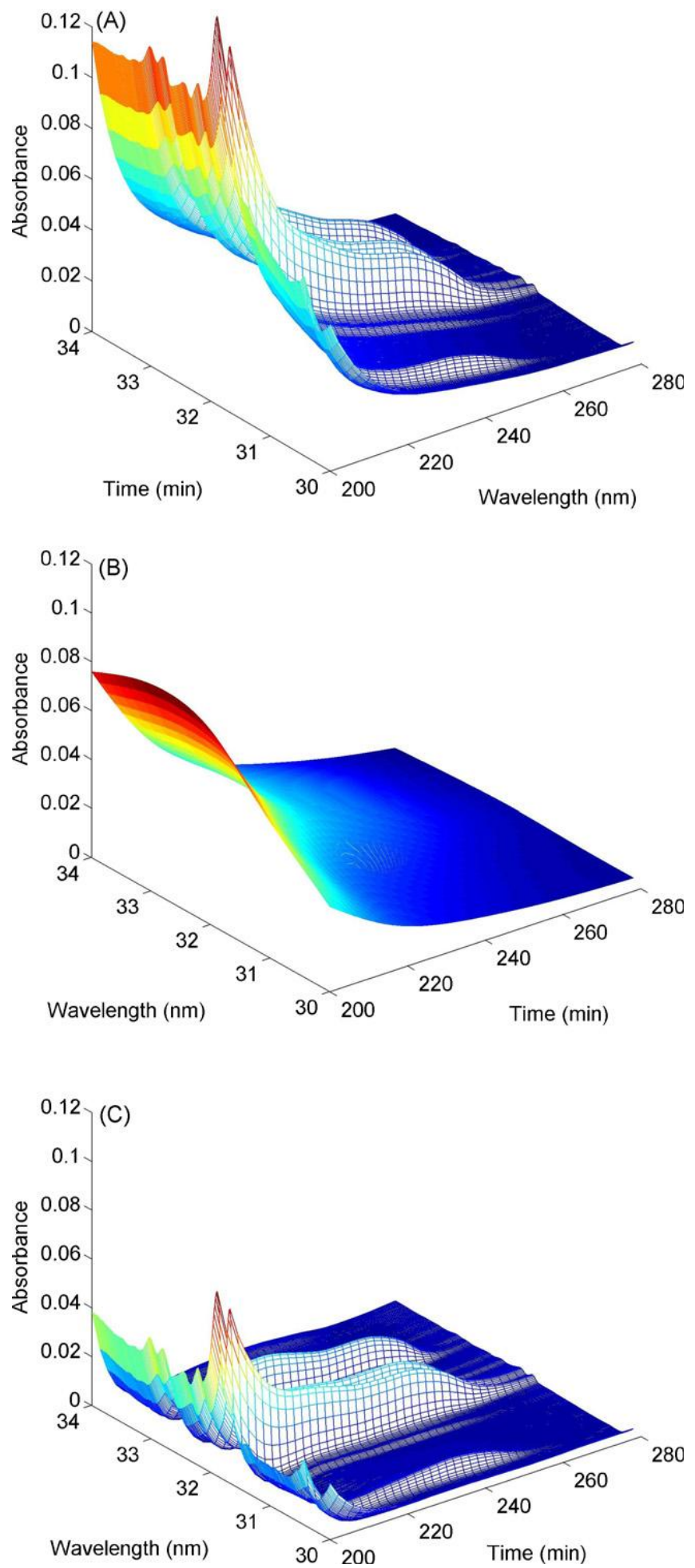

Fig. 1. (A) Landscape obtained by HPLC-DAD corresponding to a water river sample obtained after spiking the river water sample with different concentrations of the analytes (sample R1-1 of Ref. [11]) in the time region of 30-34 min. (B) Background matrix corresponding to the landscape of (A). (C) Landscape obtained by subtraction of the background to the landscape of (A). 
isocratic and gradient conditions [36-38]. These algorithms use a data set recorded from a gradient experiment without injecting any analyte. By matching characteristic absorption bands of the eluents in the chromatographic run with those of the reference data set, it is possible to select or to calculate an appropriate background spectrum for recovering the analyte spectra. Detailed descriptions of the procedure and examples of its application can be found in the former work [36]. Very recently, the algorithm has been successfully applied to the chromatographic separation of four nitrophenols [39] and these authors have published a review about the advances in isocratic and gradient liquid chromatography hyphenated on-line with infrared (LC-IR) spectrometry, placing particular emphasis on chemometric background correction and other applications of chemometric algorithms used to improve the sensitivity and the resolution of LC-IR signals [40].

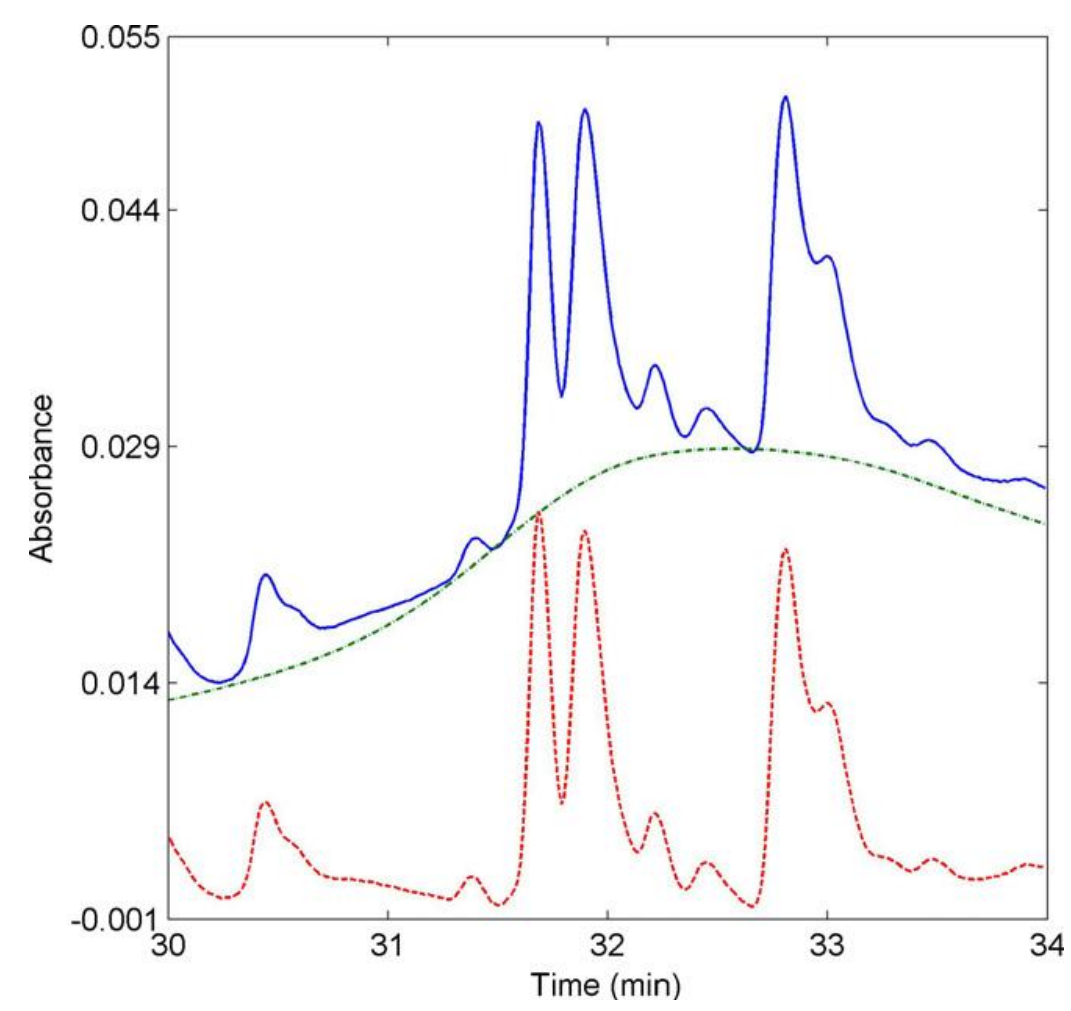

Fig. 2. Chromatogram (registered at $\lambda=245 \mathrm{~nm}$ ) of a water river sample obtained after spiking the river water sample with different concentrations of the analytes (sample R1-1 of Ref. [11]) (blue solid line), the base line calculated at the same wavelength (green point-dashed line), and the corrected chromatogram by subtraction of the base line to the original chromatogram (red dashed line). 
Ramis-Ramos and co-workers [41] presented an automatic two way background correction using cubic smoothing splines (CSS) and multivariate data analysis to two-way electropherograms, which were automatically processed, with minimal supervision by the user, in less than $2 \mathrm{~min}$. A simple background elimination method for Raman spectra, based on peak detection, smoothing, and interpolation was proposed by Baek et al. [42]. The authors postulate that since the background is usually slowly varying with respect to wavelength, they could estimate the background by eliminating significant peaks. They seek the peaks by inspecting the smoothed derivative of a given spectrum, and after clipping out the corresponding peak regions, an estimation of the background by applying a modified linear interpolation was performed.

A novel technique for removal of three-dimensional background drift in comprehensive twodimensional (2D) liquid chromatography coupled with diode-array detection (LCXLC-DAD) data was proposed by $\mathrm{Yu}$ and co-workers [43]. The authors worked on the basic idea of performing trilinear decomposition, based on the alternating trilinear decomposition (ATLD) algorithm, on the instrumental response data. A model was built taking into account the background drift as one component or factor as well as the analytes of interest, hence, the drift being explicitly included into the calibration.

\subsection{Time shift correction}

When applying second-order algorithms, especially for methods such as GRAM, PARAFAC, BLLS and U-PLS/RBL, peaks should be properly aligned in order to assure trilinearity in the data. Several approaches have been presented in the literature for synchronization of the time axes. This procedure is called warping or alignment, and as will be seen, it is a crucial subject when analyzing complex samples. If a proper alignment can be applied, the posterior data analysis would be simplified, but it has been shown that there are some cases in which shift correction can not be implemented $[12,44]$. The latter fact happens when potential interferences coelute with the analytes. In the present review, several approaches will be commented, and a brief description of the most important ones will be made.

The alignment algorithms are based on different basic philosophies. In a first group, advantage of the matrix data structure is taken: rank alignment (RA) $[45,46]$ and iterative target transformation factor analysis (ITTFA) [47,48]. In a second group, the maximum correlation between chromatograms is sought: the so-called ChromAlign algorithm [49] and correlation optimized warping (COW) [50-53]. Finally, a suitably initialized and constrained PARAFAC model can be used [44]. It should be noted that taking into account that the presence of potential interferences in 
unknown samples poses severe challenges to the above-mentioned alignment algorithms, especially for the second group of algorithms, these will not be commented.

The RA algorithm is based on the singular value decomposition (SVD) of an N/M matrix, joining the data matrices $\mathbf{N}$ and $\mathbf{M}$, where $\mathbf{N}$ is taken as reference and $\mathbf{M}$ is the matrix which has to be corrected in relation to $\mathbf{N}$. The correction is carried out by computing the residual variance (RES) while the matrix $\mathbf{M}$ is moved in relation to $\mathbf{N}$, using a pre-established number of points that can be estimated by the inspection of the chromatograms of $\mathbf{N}$ and $\mathbf{M}$. The number of significant singular values should ideally be equal to the number of species present in $\mathbf{N} \mid \mathbf{M}$. When the matrices $\mathbf{N}$ and $\mathbf{M}$ are aligned, the RES values should reach a minimum. It should be noted that problems appear when the test sample contains interferences highly overlapped with the analyte peak, and more than one minimum is obtained. In contrast, in the ITTFA algorithm the calibration and test matrices are independently decomposed into profiles and spectra, and aligned before the second-order method is applied [47]. At the top of Fig. 3 it is shown the contour plots for matrices $\mathbf{N}$ and $\mathbf{M}$ (used to build the augmented $\mathbf{N} / \mathbf{M}$ matrix) before the alignment. Matrixes $\mathbf{N}$ and $\mathbf{M}$ correspond to the determination of carbamazepine in the presence of interferences using capillary electrophoresis with DAD detector (see Ref. [54]). As can be seen in this figure, there is a difference of c.a. $1.5 \mathrm{~min}$ between the reference matrix $(\mathbf{N})$ and the one to be corrected (M). If matrix $\mathbf{M}$ is moved 64 data points in the direction indicated by the arrow, a minimum in the RES value is reached. This fact can be more clearly appreciated in Fig. 4.

Finally, the PARAFAC alignment is based on analyzing the fit of a PARAFAC model for a three-way array built with matrices $\mathbf{N}$ and $\mathbf{M}$ placed on top of each other. If potential interferents coelute with the analytes, the correct alignment of $\mathbf{M}$ with respect to $\mathbf{N}$ requires two bilinear contributions, i.e., two spectral-time retention matrices (one for the analyte and one for the interferent). On the other hand, the incorrect possibility should in principle require three bilinear components: two of them correspond to the analyte (having the same spectrum but different peaks in the time dimension), and the remaining one to the interferent. Other possibilities to correct peaks misalingned will also require three bilinear components. Therefore, a suitably initialized and restricted two component PARAFAC model will only yield a reasonable fit when the correct alignment is performed. As can be observed, this method exploits the matrix structure of the studied data in order to align the test data matrix with respect to the reference one [44]. 

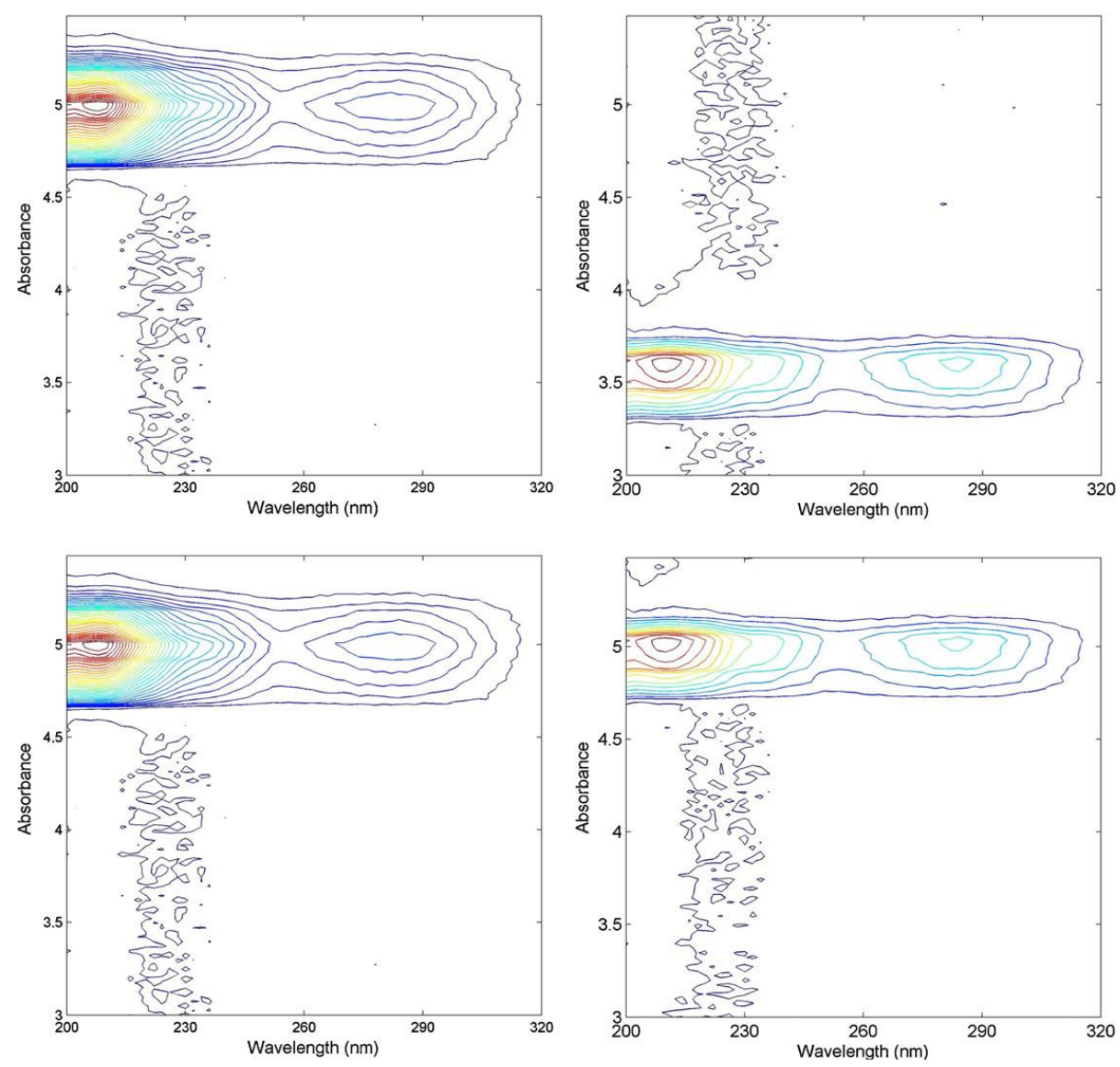

Fig. 3. Top: contour plots for matrices $\mathbf{N}$ and $\mathbf{M}$ corresponding to the determination of carbamazepine in the presence of interferences using capillary electrophoresis with DAD detector (see Ref. [53]) before the alignment. Bottom: the same matrices after alignment by shifting 64 data points matrix $\mathbf{M}$ in the direction of the arrow.

\section{Second-order algorithms}

As was mentioned above, coupling chromatography or capillary electrophoresis with either diode array, fast-scanning fluorescence or mass detectors originate second-order data. The spectroscopic response is therefore arranged as a data matrix, where each column corresponds to a wavelength (or $\mathrm{m} / \mathrm{z}$ ratio) and each row corresponds to a different time. Interestingly, in those cases in which full selectivity in the chromatographic separation is not achieved, calibration can be performed, and quantitation can be accomplished in the presence of unexpected constituents and only synthetic standards are necessary for the model development, in those cases in which there is not matrix effect (see above). Thus, a data set for several samples (unknowns plus standards) can be 
conveniently arranged into different modes: (a) to build a threeway array (PARAFAC, PARAFAC2, GRAM, DTLD, APTLD, SWATLD and N-PLS), (b) to arrange the second-order data set into an augmented matrix, as is regularly done in MCR-ALS; (c) to vectorize the higher-order sample data and then employ first-order multivariate methods (the unfolded variants of PLS or ANN); (d) to combine the unfolded variants with the RBL procedure in order to exploit the secondorder advantage (BLLS, U-PLS/RBL and ANN/RBL); and (e) to combine the three-way array of NPLS with the RBL procedure in order to exploit the second-order advantage, as this property is not fulfilled by this latter algorithm (see Table 1). All of these algorithms allow for the development of the so-called second-order multivariate calibration methods.

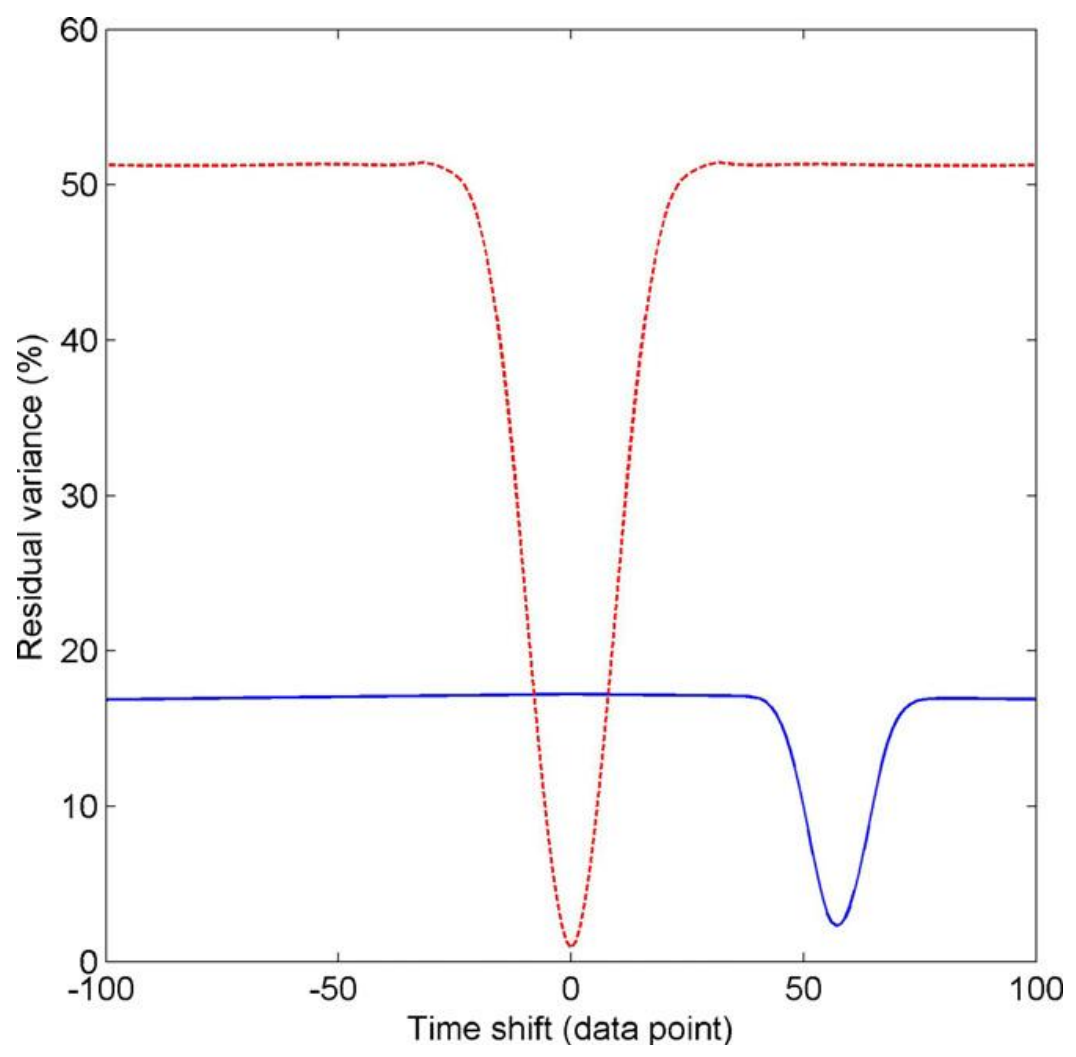

Fig. 4. Residual variance computed for the augmented N/M matrix when shifting $\mathbf{M}$ (solid blue line). The dashed red line shows the residual variance computed for an augmented $\mathbf{N} / \mathbf{N}$ matrix. 
Table 1. Algorithms employed to model second-order chromatographic data with calibration purposes

\begin{tabular}{lcc}
\hline Application & Algorithm & References \\
\hline Building three-way arrays & PARAFAC & {$[10,28,47,48,58,64]$} \\
& PARAFAC2 & {$[9]$} \\
& GRAM & {$[47,48,82,84,85]$} \\
& DTLD & {$[15]$} \\
& APTLD & {$[57]$} \\
Arranging the second-order data $\quad$ set $\quad$ into & N-PLS & {$[16]$} \\
augmented matrix & MCR-ALS & {$[9,10,12,47,48,59-63,65-71,83,85]$} \\
$\begin{array}{l}\text { Combining the unfolded variants with the RBL } \\
\text { procedure in order to exploit the second-order } \\
\text { advantage }\end{array}$ & BLLS & {$[28,58]$} \\
$\begin{array}{l}\text { Combining the three-way array of N-PLS with the } \\
\text { RBL procedure in order to exploit the second-order } \\
\text { advantage }\end{array}$ & N-PLS/RBL & {$[59,60]$} \\
\hline
\end{tabular}

From an analytical point of view, the main object of using second-order data is to assure exploiting the second-order advantage. For this purpose, two alternative ways are possible [6]: (a) data for the test sample has an influence on the regression coefficients leading to prediction (PARAFAC, PARAFAC2, GRAM, DTLD, APTLD, SWATLD and MCR-ALS) or (b) calibration is first performed using only training data, with the test sample leading to sample-specific regression coefficients in a subsequent step (BLLS, U-PLS/RBL, NPLS/RBL and ANN/RBL).

Comprehensive information about the different second-order algorithms can be found in the pertinent literature [13-21]. In addition, complete reviews were presented with a wide range of applications to second-order data (including chromatography) [6-8]. Thus, in this review we will revise the most recent and important applications, especially in those cases in which complex samples were analyzed and overlapping peaks had to be solved because of incomplete selectivity due to insufficient separation among analytes or the presence of unexpected components in the sample.

As was previously discussed, when data are not trilinear, there exist algorithms which can overcome this problem, and calibration can be performed without pre-treatment of the data (see above). The most popular one is MCR-ALS [19], which resorts to the mathematical resource of matrix augmentation. Alternatively, several applications of PARAFAC2 have been recently presented, which allows for separate time profiles in each experimental sample [55]. On the other hand, when data are conveniently pre-treated, good results have been presented by applying GRAM, PARAFAC or RBL based algorithms [6,10,44,56]. 


\subsection{UV spectroscopy with diode-array detection}

$\mathrm{Li}$ et al. presented a method based on modeling LC-DAD data with the alternating trilinear decomposition (ATLD) algorithm for the quantitative analysis of levodopa, carbidopa and methyldopa in human plasma samples. Although the spectra of these analytes were similar and interferents coeluted with the analytes studied in biological samples, good recoveries of the analytes were obtained, with additional benefits like decreasing times of analysis and less solvent consumption [57].

Braga et al. presented the simultaneous determination of five pesticides and two metabolites in wine samples by HPLCDAD, using the second-order advantage. The authors compared two chromatographic methods, which involve either isocratic or gradient elution. Due to loss of trilinearity, an appropriate preprocessing method was necessary to correct the effects of time shifts, baseline variations and background. BLLS yielded results that were of the same quality as PARAFAC in five cases, but in two other situations only PARAFAC enabled analyte quantitation [28]. In a posterior work, these authors presented a variable selection methodology based on genetic algorithm to improve the results [58].

Several methods were developed in our group to be applied in the quantitation of diverse analytes in environmental samples by modeling liquid chromatography data with MCR-ALS and UPLS/RBL algorithms. Firstly, eight tetracycline antibiotics were determined in effluent wastewater, solving matrix effects and exploiting the second-order advantage [59,60]. Afterward, the determination of anti-inflammatory and antiepileptic drugs in river and wastewater by solid-phase microextraction and liquid chromatography diode-array detection with MCR-ALS was presented [61]. The following study included 11 pharmaceuticals which were quantitated in river water by column switching of large sample volumes and HPLC-DAD, modeling with MCR-ALS [62]. Additional works involved the determination of dyes in beverages reaching a considerable reduction of the analysis time [12], and the resolution of fully overlapped capillary electrophoresis peaks applied to the quantitation of carbamazepine in human serum in the presence of several interferences [63]. It should be noticed that, owing to the complete overlapping among three of the analytes, the only algorithm capable of resolve this task was MCR-ALS.

In a study presented by Vosough et al., a second-order calibration strategy for the simultaneous determination of aflatoxins B1, B2, G1 and G2 in pistachio nuts in the presence of matrix interferences has been developed combining HPLC-DAD and PARAFAC. Sample preparation was based on solvent extraction followed by solid-phase extraction. Since the sample preparation procedure was not selective to the analytes of interest, exploiting second order advantage to obtain concentrations of individual analytes in the presence of uncalibrated interfering compounds was 
necessary. Appropriate pre-processing steps were applied to correct background signals and the effect of retention time shifts. The proposed method presented advantages like using a low-cost SPE step, a unique and simple isocratic elution program for all samples and a calibration transfer for saving both chemicals and time of analysis [64].

Finally, MCR-ALS was evaluated in the analysis of nine phenolic acids, both in standards mixture samples and in strawberry juice samples by LC-DAD. Chromatographic co-elution problems either because of unknown matrix interferences or because of the increase of organic modifier to reduce chromatographic analysis times were investigated. Results obtained in the resolution and quantitation of phenolic acids in standards mixture samples and strawberry samples showed that the proposed MCR-ALS approach reduces analysis times and solvent expenses and improves determinations in case of strong co-elution [65].

A reduced number of CE-DAD applications can be found in the literature, most of them based on MCR-ALS modeling. This fact is due to this technique generally originates data which are not

trilinear. Several ebrotidines metabolites were analyzed and pure spectra and electrophoretic profiles were conveniently extracted by MCR-ALS in a study presented by Sentellas et al. [66]. In addition, Li and co-workers presented several strategies to enhance the quantitation of several analytes combining CE-DAD data and MCRALS [67-69]. Finally, we presented a challenging application in which three analytes, whose peaks were totally overlapped, could be determined in serum samples with acceptable analytical figures of merit [54].

\subsection{Fast-scanning fluorescence spectroscopy (FS-FS) detection}

It is important to notice that a reduced number of applications in this area have been presented. In a study carried out by Cañada- Cañada et al., different second-order algorithms were compared (PARAFAC, N-PLS/RBL and MCR-ALS) for the analysis of four fluoroquinolones in aqueous solutions, including some human urine samples. Data were measured in a short time with a chromatographic system operating in the isocratic mode. The detection system consisted of a fastscanning spectrofluorimeter obtaining second-order data matrices containing the fluorescence intensity as a function of retention time and emission wavelength. Interestingly, although the analytes presented overlapped profiles, it was not necessary to apply an elution gradient, and thus significantly reducing both the experimental time and complexity [10].

In a very recent and interesting application presented by Bortolato et al., the analysis of 10 polycyclic aromatic hydrocarbons (PAHs) was performed. The goal of the work was the successful resolution of a system even in the presence of real interferences. Second-order HPLC-FS-FS data matrices were obtained in a short time with a chromatographic system operating in isocratic mode. 
The difficulties in aligning chromatographic bands in complex systems were discussed. Two second-order calibration algorithms which do not require chromatographic alignment were selected (MCR-ALS and PARAFAC2), the superiority of MCR-ALS to successfully resolve this complex system [9], being demonstrated.

Pere-Trepat et al. applied MCR-ALS to solve co-elution problems in liquid chromatography with DAD and MS detection. Interestingly, a MCR-ALS property allowing the fusion of both detector signals improved results versus those obtained using only one of the two detector signals. Wavelet transform had to be applied to MS data before its fusion with DAD data, which further facilitated the resolution and quantitation of the coeluted compounds under study, besides a decrease of time of analysis. Mixtures of biocide compounds in standard mixtures and in environmental samples (sediment and wastewater samples) were analyzed with acceptable quantitation errors considering the complexity of the samples [70].

Finally, in an application to a metabonomic study, a chemometric strategy based on MCR-ALS applied to LC-MS in the scan mode was developed by our group to perform a metabonomic study in tomato fruits following treatment with carbofuran. The methodology proved to be adequate for the detection of unintended stress effects due to the previous treatment with this pesticide. MCRALS was performed on augmented matrices built with the data obtained from treated and nontreated samples through the sampling time. By applying this strategy the concentration and spectra profiles of the main components were obtained from samples treated with pesticide as well as from blank samples, showing how they vary with time after plants treatment with the pesticide. In addition, a simple resolved mass spectrum was obtained corresponding to the peaks of a particular component in all matrices, thus avoiding ambiguity in the compound identity assignment. Different time profiles were found for some metabolites in treated and non-treated samples, which demonstrate that the presence of pesticide causes changes through time in the behavior of certain endogenous tomato metabolites as a result of physiological stress [71].

\section{Variations in the analytical response}

Generally, quantitation of analytes in real samples is performed using calibration models built with standards prepared in pure solvent. However, in some cases it can be observed that the analyte response in real samples is different from the one obtained for analytes prepared in pure solvents. Apart of background and additive interferences, the main causes of the variation in the analyte signal are due to the phenomenon known as matrix effect and to the implementation of treatment steps prior to the measurement of analyte, such as extraction or clean-up steps. 
In multivariate (as in univariate) calibration methodologies, the correction of changes in the analyte signal of real samples requires the application of different strategies depending of the type of phenomena that causes these changes.

Three classes of strategies have been proposed to correct the matrix effect: (a) based on sample handling, e.g., selective extraction, effective sample clean-up after extraction or improvement of the chromatographic separation, (b) based on the reduction of matrix components injected onto the chromatographic system, which can be performed by simply injecting smaller volumes in the chromatographic system or by diluting the sample extract; and (c) based on suitable calibration approaches, e.g., the use of internal standards, matrix-matched standards and standard addition of each analyte into each sample.

Strategies included in the first group require an additional effort, are rather time consuming [72], in some cases the problem persists, and, in addition, they can cause losses of the analytes related to extraction and/or clean-up process.

On the other hand, each of the approaches used to reduce matrix components loaded on the detector involves a lack of sensitivity [73].

The third group of strategies also shows some drawbacks. Thus, matrix-matched standards are often used to correct the matrix effect in spite of the great drawback which involves the availability of real samples not containing the analytes of interest (i.e., real blank samples). In addition, as matrix effect is attributed to organic and/or inorganic components of the sample co-eluting with the analytes and interfering during the detection process, it is matrix dependent being rather variable between samples, in such a way that matrix effect in a series of samples can also be highly variable and difficult to predict [74]. Therefore, errors in the prediction should be expected when using only a matrix-matched curve for the quantitation of analytes in all different sample matrices.

According to Benijts et al. [75] the best way to tackle matrix effect is to use appropriate internal standards, being preferred an isotopically labeled one, even though they are not regularly available and alternatively structural analogues are used [76]. On the other hand, the internal standard had to elute close to the compound of interest, showing similar behavior in the detector, in such a way that more than one of them should be used [77]. It is generally observed that using internal standards derives in significant enhancement of certain analytical figures of merit such as precision, linearity and accuracy.

The standard addition methodology implies the addition of increasing amounts of a standard of the analytes to several portions of sample $(n=4-6)$ to build the calibration curve for the quantitation of the analytes in each sample. Even though their use requires a great amount of sample and, in addition, it leads to a significant increase in analysis and processing time, as one calibration 
curve must be built for analyzing each sample, nowadays it is the only fairly effective methodology dealing with matrix effect. An interesting example of calibration using internal standard with second-order data for an experiment with CE-DAD data was presented by Zhang and Li et al. [69].

Finally, the standardization of the analyte signal has been currently used to compensate changes in the analytical signal, which are due to the implementation of treatment steps prior to the measurement of the analytes, such as extraction or clean-up. The most widely used methodology for standardization is piecewise direct standardization (PDS) [78], which consist of relating the response of a sample measured in a "situation A" to its response obtained in a "situation B". This relationship is described by the transformation matrix $\mathbf{F t}$, according to

$$
X_{B}=X_{A} F_{t}
$$

where $\mathrm{XA}$ and $\mathrm{XB}$ are the response matrices of the standardization samples obtained from the $\mathrm{A}$ and $\mathrm{B}$ conditions, respectively. PDS builds a multivariate model between the response $\mathbf{r}$ of a sample measured at the jth wavelength in the situation $\mathrm{A}$ and the corresponding window (a selected region) of the response obtained on situation $\mathrm{B}$ :

$r_{j}=R_{j} b_{j}$

where $\mathbf{R}_{\mathrm{j}}$ is the localized response matrix of the transfer samples and $\mathbf{b}_{\mathrm{j}}$ is the vector of transformation coefficients for the jth wavelength. The regression vectors calculated for each window in the data are then assembled to form a banded diagonal matrix $\mathbf{F}_{\mathrm{t}}$, according to

$$
F_{t}=\operatorname{diag} \mathbf{Q}_{1}^{T},_{2}^{T}, \ldots . . b_{j}^{T}, \ldots \ldots . . b_{k}^{T}
$$

where $\mathrm{k}$ is the number of wavelengths. The response of any unknown sample (xs) can then be standardized according to the equation:

$$
x_{s}=x^{T} F
$$

All these approaches have been successfully applied to univariate data, but recently, a number of papers have been published, which report the application of some of the above-mentioned methodologies in combination with second-order multivariate algorithms, with satisfactory results $[8,59,60,64]$.

\subsection{Strategies based on calibration approaches (standard addition and internal standard)}

In analytical chemistry, the standard addition method has been employed to perform quantitative analysis in situations where external calibration is not feasible, basically to overcome the problem of changing matrix effects [79]. Matrix effect occurs when the sample contains organic and 
inorganic components that not give a response but coelute with the target analytes [80] causing variations in their responses, thus affecting sensitivity and accuracy of the analytical method and therefore producing relative systematic errors. Matrix effect occurs especially in chromatographic techniques coupled with mass spectrometric detection (MS) due to a suppression of the ionization efficiency of the analytes in the presence of co-eluting substances and coupled with UV or fluorescence detectors due to a suppression or enhancement of the signal as the result of the interactions of the analytes with other matrix components which modify their absorptive or emissive properties. As stated above, this behavior may be rather variable between samples, in such a way that matrix effects in a series of samples can also be highly variable and difficult to predict [81]. Therefore quantitation should be carried out by applying the standard addition method, as this methodology assures that the standards used in the calibration step are undergone to the same effect that the analytes contained in the analyzed samples.

The hardly difficult issue related to the analysis of highly complex samples may be handled using second (and higher) order data from hyphenated techniques coupled to multivariate calibration algorithms involving the second-order advantage in combination with the standard addition methodology. The second-order advantage allows accurate quantitation of multiple analytes using calibration samples containing multiple chemical components without knowledge of the interfering chemical components whereas standard addition copes with matrix effect. This methodology has been widely used with spectroscopic data whereas a less number of applications have been published using chromatographic techniques.

\subsubsection{Liquid chromatography}

Gimeno et al. [82] applied GRAM combined with standard addition to quantify polycyclic aromatic compounds in marine sediments by liquid chromatography with diode-array detection. This second-order algorithm was applied to three-way data obtained using a program gradient not requiring the complete separation of analytes and was compared with a previously optimized univariate method for these analytes in the same real samples with longer analysis time. The similarity of the results obtained using GRAM with those obtained with univariate calibration showed that second-order methodology is advantageous in situations in which the analytes cannot be completely separated or the analysis would be time consuming if complete separation is achieved. In both cases, they found the added advantage that the standard addition method succeed the quantitation of target analytes in samples with matrix effect.

Recently, in our group the second-order algorithm MCR-ALS was applied in combination with standard addition calibration for the determination of drugs in environmental water samples by LCDAD [61,62]. In a first work, eight pharmaceuticals were determined in wastewaters samples [59] 
containing compounds interfering with the analytes of interest with a variable effect between samples, in addition to matrix effect. MCR-ALS coped with the problems of overlapped interferences whereas the standard addition corrected the different behavior of the wastewater matrix in the response of the analytes. Then, MCR-ALS was applied in combination with the standard addition calibration method to deal with overlapping peaks and systematic (additive) and proportional (matrix effect) errors in the determination of eleven pharmaceuticals by LC-DAD in river water samples [62].

Finally, Tauler and co-workers [83] applied different approaches in combination with MCR.ALS for the quantitation of six biocide compounds in mussel samples, undergone to co-elution and to matrix effect in LC-MS. Among the three calibration strategies used (external calibration, standard addition and internal standard), multivariate extension of the standard addition method using MCRALS provided an improvement in the results which was increased when internal standard was additionally used on the same mussel matrix sample.

\subsubsection{Gas chromatography}

The application of second-order algorithms to three-way data generated in gas chromatography (GC) using standard addition is more limited that in LC. In our knowledge, only two papers reported the application of second-order methodologies in combination with standard addition to quantify analytes in complex samples that causes matrix effect in the GC responses. In this sense, Fraga et al. [84] used GRAM with two-dimensional GC(GC-GC) coupled to a flame ionization detector (FID) for the determination of aromatic isomers in a jet fuel. The application of GRAM allowed for the separation of the overlapped $\mathrm{GC} \times \mathrm{GC}$ peaks, whereas the standard addition calibration corrected changes in peak widths and retention times between samples and standards.

Finally, Vosough and Salemi [85] applied two second-order calibration methods (GRAM and MCR-ALS) on standard addition data matrices obtained by GC-MS, to characterize and quantify fatty acids in fish oil, and the results obtained were compared. As trilinearity is the essential requirement for implementing GRAM a retention algorithm was applied with similar results to those obtained by MCR-ALS. In both cases, the combination of second-order calibration methods with standard addition calibration showed the great potential of these approaches as an efficient way for solving matrix effect in GC-MS in complex samples.

\subsection{Standardization}

There are several reasons to implement transference of models (standardization), which include the following examples: (a) need to transport a calibration model previously built on the first instrument to another; (b) changes in the instrument over time; (c) variation between samples from 
different production batches; and (d) situations when necessary sample treatments such as extraction or clean-up steps are carried out [86]. The latter case was observed with different extraction techniques such as solid-phase extraction (SPE) [59,60,64] and solid-phase microextraction (SPME) [61]. These sample treatments are necessary to increase sensitivity of the analytical methods when analytes are present at ultratrace levels in the samples being analyzed. However, in some cases, the treatment step causes partial losses or changes in the response signal of analytes. This drawback would be overcome by undergoing the analytical standards to the SPE step, but this option demands considerable both time and cost, which makes advisable the use of transference models. By applying standardization, only a reduced number of the whole calibration samples in the real situation is necessary for building a useful model to predict new real sample signals.

PDS was applied to correct the breakthrough effect observed for the most polar analytes after the SPE step in a method developed for the determination of eight tetracyclines in effluent wastewater samples by solid-phase extraction (SPE) and LC-DAD using MCR-ALS [60] and U-PLS/RBL [61] algorithms. The five first eluting compounds were partially lost during the pre-concentration step and this drawback was overcome by transferring sample signals obtained from standards prepared in pure solvent to signals after SPE and then, unknown samples were predicted with models built with the former.

Very recently, Vosough et al. [64] used PDS combined with PARAFAC algorithm to quantify four alfatoxins in extract of pistachio nuts by LC-DAD, in the presence of interferences remaining after SPE. PARAFAC dealt with the problem caused by non-modeled interferences and a transferred calibration data set, obtained from standardization of solvent based calibration data, was used in the prediction step. Using these approaches, the cost per analysis was also significantly reduced.

PDS combined with MCR-ALS was also applied in the determination of seven non-steroidal anti-inflammatory drugs and one anticonvulsant in river and wastewater by SPME-LC-DAD [61]. In SPME, standards and samples must be processed in the same way, which increase the error in handling, leading to the fact that the main source on uncertainty is that associated with the calibration step [87], along with an increase in the time spent in the calibration step. In order to avoid the pre-concentration of the calibration standards, PDS was applied to transform the signal obtained from direct injection of standards in the LC-DAD system to the signals obtained by standards prepared in Milli-Q water and subjected to the SPME step. In the case of river water, PDS became a useful tool in reducing the number of standard samples which must be undergone to SPME for calibration, thus allowing quantifying pre-concentrated river water samples. 


\section{Conclusions}

Analytical chemistry involves the analysis of complex samples which currently needs sophisticated instrumentation and a number of handling sample steps to accomplish the goal. The use of powerful mathematical tools for data treatment is able to improve the results, in addition to save time and cost in the total analytical method.

Thus, background correction (including baseline drift and additive interferences) greatly improves spectral and elution profiles, allowing the resolution and quantitation of analytes in complex samples using second-order algorithms. In addition these algorithms are useful when trilinearity is not accomplished by the data and time shift correction is not necessary.

Even though the drawbacks inherent to the standard addition calibration method, this approach is a fairly effective methodology dealing with matrix effect, which has been successfully applied in combination with second-order algorithms for the analysis of complex samples.

The standardization of second-order data sets allows to correct changes in the analytical signal after sample pre-treatments in addition to reduce the number of standard samples that must be prepared for calibration when they must be undergone to the same pre-treatment that the samples.

\section{Acknowledgments}

The authors are grateful to CONICET (PIP 112-200801-02988), ANPCyT (PICTO 55905 No. 35124), Universidad Nacional del Litoral (CAI +D 12-65) and MINISTERIO DE CIENCIA Y TECNOLOGIA (Proyecto CTQ2007-65954). M.J.C. and H.C.G. are members of the research career from CONICET.

\section{References}

[1] G. Hanrahan, R. Montes, F.A. Gomez, Anal. Bioanal. Chem. 390 (2008) 169.

[2] M.W. Dong, Modern HPLC for Practicing Scientists, Wiley, Synomics Pharmaceutical Services, LLC, Wareham, MA, 2006.

[3] A. Kloepfer, J.B. Quintana, T. Reemstsma, J. Chromatogr. A 1067 (2005) 153.

[4] M. Daszykowski, B. Walczak, TrAC Trends Anal. Chem. 25 (2006) 1081.

[5] K.S. Booksh, B.R. Kowalski, Anal. Chem. 66 (1994) 782A-791A.

[6] G.M. Escandar, N.M. Faber, H.C. Goicoechea, A.T.A. Muñoz de la Peñaa, A.C. Olivieri, R.J. Poppi, TrAC Trends Anal. Chem. 26 (2007) 752.

[7] V. Gomez, M.P. Callao, Anal. Chim. Acta 627 (2008) 169.

[8] M. Martinez Galera, M.D. Gilgarcia, H.C. Goicoechea, TrAC Trends Anal. Chem. 26 (2007) 1032.

[9] S.A. Bortolato, J.A. Arancibia, G.M. Escandar, Anal. Chem. 81 (2009) 8074. 
[10] F. Cañada-Cañada, J.A. Arancibia, G.M. Escandar, G.A. Ibañez, A. Espinosa Mansilla, A. Muñoz de la Peña, A.C. Olivieri, J. Chromatogr. A 1216 (2009) 4868.

[11] M. Martinez Galera, M.D. Gil Garcia, M.J. Culzoni, H.C. Goicoechea, J. Chromatogr. A 1217 (2010) 2042.

[12] M.J. Culzoni, A.V. Schenone, N.E. Llamas, M. Garrido, M.S. Di Nezio, B.S. Fernandez Band, H.C. Goicoechea, J. Chromatogr. A 1216 (2009) 7063.

[13] E. Sanchez, B.R. Kowalski, Anal. Chem. 58 (1986) 496.

[14] E. Sanchez, B.R. Kowalski, J. Chemom. 1 (1990) 29.

[15] P.D. Wentzell, S.S. Nair, R.D. Guy, Anal. Chem. 73 (2001) 1408-1415.

[16] Z.P. Chen, H.L. Wu, J.H. Jiang, Y. Li, R.Q. Yu, Chemom. Intell. Lab. Syst. 52 (2000) 75.

[17] A.L. Xia, J.L. Wu, D.M. Fang, Y.J. Ping, L.Q. Hu, R.Q. Yu, J. Chemom. 19 (2005) 65.

[18] R. Bro, Chemom. Intell. Lab. Syst. 38 (1997) 149.

[19] R. Tauler, Chemom. Intell. Lab. Syst. 30 (1995) 133.

[20] M. Linder, R. Sundberg, Chemom. Intell. Lab. Syst. 42 (1998) 159.

[21] S. Wold, G.P.K. Esbenesen, J. Ohman, J. Chemom. 1 (1987) 41.

[22] A.C. Olivieri, J. Chemom. 19 (2005) 615.

[23] A. de Juan, R. Tauler, Anal. Chim. Acta 500 (2003) 195.

[24] R. Bro, Doctoral Thesis, University of Amsterdam, The Netherlands, 1998.

[25] C.B. Zachariassen, J. Larsen, F. van den Berg, R. Bro, A. de Juan, R. Tauler, Chemom. Intell. Lab. Syst. 83 (2006) 13.

[26] J.M.M. Leitao, J.C.G. Esteves da Silva, Chemom. Intell. Lab. Syst. 89 (2007) 90.

[27] M.D. Gil Garcia, M.J. Culzoni, M.M. De Zan, R. Santiago Valverde, M. Martinez Galera, H.C. Goicoechea, J. Chromatogr. A 1179 (2008) 106.

[28] J.W.B. Braga, C.B.G. Bottoli, I. Jardim, H.C. Goicoechea, A.C. Olivieri, R.J. Poppi, J. Chromatogr. A 1148 (2007) 200.

[29] T.L. Cecil, S.C. Rutan, J. Chromatogr. A 556 (1991) 495.

[30] Y.-Z. Liang, O.M. Kvalheim, A. Rahmani, R.G. Brereton, Chemom. Intell. Lab. Syst. 18 (1993) 265.

[31] P.H.C. Eilers, Anal. Chem. 76 (2004) 404.

[32] P.H.C. Eilers, I.D. Currie, M. Durban, Comp. Stat. Data Anal. 50 (2006) 61.

[33] K. Kaczmarek, B. Walczak, S. de Jong, B.G.M. Vandginste, Acta Chromatogr. 15 (2005) 82.

[34] K. Istva’n, R. Rajko`1, G. Keresztury, J. Chromatogr. A 1104 (2006) 154.

[35] T. Skov, J.C. Hoggard, R. Bro, R.E. Synovec, J. Chromatogr. A 1216 (2009) 4020.

[36] G. Quintas, B. Lendl, S. Garrigues, M. de la Guardia, J. Chromatogr. A 1190 (2008) 102. 
[37] G. Quintas, J. Kuligowski, B. Lendl, Anal. Chem. 81 (2009) 3746.

[38] J. Kuligowski, G. Quintas, S. Garrigues, M. de la Guardia, Talanta 77 (2008) 779.

[39] J. Kuligowski, G. Quintas, S. Garrigues, M. de la Guardia, Talanta 80 (2010) 1771.

[40] J. Kuligowski, G. Quintas, S. Garrigues, M. de la Guardia, B. Lendl, TrAC Trends Anal. Chem. 29 (2010) 544.

[41] V. Bernabe-Zafon, J.R. Torres-Lapasio, S. Ortega-Gadea, E.F. Simo-Alfonso, G. RamisRamos, J. Chromatogr. A 1065 (2005) 301.

[42] S.-J. Baek, A. Park, J. Kim, A. Shen, J. Hu, Chemom. Intell. Lab. Syst. 98 (2009) 24.

[43] Y. Zhang, H.-L. Wu, A.-L. Xia, L.-H. Hu, H.-F. Zou, R.-Q. Yu, J. Chromatogr. A 1167 (2007) 178.

[44] S.A. Bortolato, J.A. Arancibia, G.M. Escandar, A.C. Olivieri, Chemom. Intell. Lab. Syst. 101 (2010) 30 .

[45] B.J. Prazen, R.E. Synovec, B.R. Kowalski, Anal. Chem. 70 (1998) 218.

[46] K.J. Johnson, B.J. Prazen, D.C. Young, R.E. Synovec, J. Sep. Sci. 27 (2004) 410.

[47] E. Comas, R.A. Gimeno, J. Ferre, R.M.Marce, F. Borrull, F.X. Rius, Anal. Chim. Acta 470 (2002) 163.

[48] E. Comas, R.A. Gimeno, J. Ferre, R.M. Marce, F. Borrull, F.X. Rius, J. Chromatogr. A 1035 (2004) 195.

[49] R.G. Sadygov, F.M. Maroto, A.F.R. Huhmer, Anal. Chem. 78 (2006) 8207.

[50] N.P.V. Nielsen, J.M. Carstensen, J. Smedsgaard, J. Chromatogr. A 805 (1998) 17.

[51] T. Skov, F. van den Berg, G. Tomasi, R. Bro, J. Chemom. 20 (2006) 484.

[52] G. Tomasi, F. van den Berg, C. Andersson, J. Chemom. 18 (2004) 231.

[53] T. Skov, J.C. Hoggard, R. Bro, R. Synovec, J. Chromatogr. A 1216 (2009) 4020.

[54] L. Vera Candioti, A.C. Olivieri, H.C. Goicoechea, Electrophoresis 29 (2008) 4527-4537.

[55] R. Bro, Doctoral Thesis, University of Amsterdam, The Netherlands, 1998.

[56] M.D. Gil Garcia, M.J. Culzoni, M.M. De Zan, R. Santiago Valverde, M. Martinez Galera, H.C. Goicoechea, J. Chromatogr. A. 1179 (2008) 106.

[57] S.-F. Li, H.-L. Wu, Y.-J. Yu, Y.-N. Li, J.-F. Nie, H.-Y. Fu, R.-Q. Yu, Talanta 81 (2010) 805.

[58] R.L. Carneiro, J.W.B. Braga, C.B.G. Bottoli, R.J. Poppi, Anal. Chim. Acta 595 (2007) 51.

[59] M.M. De Zan, M.D. Gil Garcia, M.J. Culzoni, G.G. Siano, H.C. Goicoechea, M. Martinez Galera, J. Chromatogr. A 1179 (2008) 106.

[60] M.D. Gil Garcia, M.J. Culzoni, M.M. De Zan, R. Santiago Valverde, M. Martinez Galera, H.C. Goicoechea, J. Chromatogr. A 1179 (2008) 115. 
[61] M.D. Gil Garcia, F. Cãnada Cãnada, M.J. Culzoni, L. Vera-Candioti, G.G. Siano, H.C. Goicoechea, M. Martinez Galera, J. Chromatogr. A 1216 (2009) 5489.

[62] M. Martinez Galera, M.D. Gil Garcia, M.J. Culzoni, H.C. Goicoechea, J. Chromatogr. A. 1217 (2010) 2042.

[63] L. Vera-Candioti, M.J. Culzoni, A.C. Olivieri, H.C. Goicoechea, Electrophoresis 29 (2008) 4527.

[64] M. Vosough, M. Bayat, A. Salemi, Anal. Chim. Acta 663 (2010) 11.

[65] S. Mas, G. Fonrodona, R. Tauler, J. Barbosa, Talanta 71 (2007) 1455.

[66] S. Sentellas, J. Saurina, S. Hernandez-Cassou, M.T. Galceran, L. Puignou, Anal. Chim. Acta 431 (2001) 49.

[67] H. Li, F. Zhang, J. Havel, Electrophoresis 24 (2003) 3107.

[68] F. Zhang, Y. Chen, H. Li, Electrophoresis 28 (2007) 3674.

[69] F. Ahang, H. Li, Chemom. Intell. Lab. Syst. 82 (2006) 184.

[70] E. Pere-Trepat, R. Tauler, J. Chromatogr. A 1131 (2006) 85.

[71] I. Sanchez Perez, M.J. Culzoni, G.G. Siano, M.D. Gil Garcia, H.C. Goicoechea, M. Martinez Galera, Anal. Chem. 81 (2009) 8335.

[72] M. Stueber, T. Reemtsma, Anal. Bioanal. Chem. 378 (2004) 910.

[73] D. Heller, Rapid Commun. Mass Spectrom. 21 (2007) 644.

[74] A. Koepfler, J.B. Quintana, T. Reemstsma, J. Chromatogr. A 36 (2006) 163.

[75] T. Benijts, R. Dams, W. Lambert, A. De Leenheer, J. Chromatogr. A 1029 (2004) 153.

[76] B.K. Choi, D.M. Hercules, A.I. Gusev, Fresenius J. Anal. Chem. 369 (2001) 370.

[77] S. Wang, M. Cyronak, E. Yang, J. Pharm. Biomed. Anal. 43 (2007) 701.

[78] Y.D. Yang, D.J. Veltkamp, B.R. Kowalski, Anal. Chem. 63 (1991) 2750.

[79] D.C. Harris, Quantitative Chemical Analysis, WH Freeman and Company, New York, 1999.

[80] T. Reemtsma, TrAC Trends Anal. Chem. 20 (2001) 533.

[81] A. Kloepfer, J.B. Quintana, T. Reemtsma, J. Chromatogr. A 1067 (2005) 153.

[82] R.A. Gimeno, E. Comas, R.M.Marce, J. Ferre, F.X. Rius, F. Borrull, Anal. Chim. Acta 498 (2003) 47.

[83] E. Pere-Trepat, S. Lacorte, R. Tauler, Anal. Chim. Acta 595 (2007) 228.

[84] C.G. Fraga, B.J. Prazen, R.E. Synovec, Anal. Chem. 72 (2000) 4154.

[85] M. Vosough, A. Salemi, Talanta 73 (2007) 30.

[86] R.N. Feudale, N.A. Woody, H. Tan, A.J. Myles, S.D. Brown, J. Ferre, Chemom. Intell. Lab. Syst. 64 (2002) 181. 
[87] L. Vera-Candioti, M.D. Gil Garcia, M. Martinez Galera, H.C. Goicoechea, J. Chromatogr. A 1211 (2008) 22. 